\title{
Performance of the LHCb Detector during 2010-2011 data taking
}

\author{
Martin van Beuzekom ${ }^{* \dagger}$ \\ Nikhef, Science Park 105, 1098 XG Amsterdam, The Netherlands \\ E-mail: martinb@nikhef.nl
}

The status and performance of the LHCb detector during the physics LHC physics run is described. The LHCb detector has a number of notable features including: 12 micron resolution in the transverse plane on 30-35 track primary vertices, pion and kaon separation from 1 to 100 $\mathrm{GeV}$, and $1 \mathrm{MHz}$ full readout of all sub-systems. The detector is being operating above its design luminosity and is comprised of a silicon vertex detector, silicon and straw-tube tracking systems, ring imaging Cherenkov particle identification systems, electromagnetic and hadronic calorimetry, and muon systems. Hardware and software based trigger levels are utilised to efficiently select leptonically and hadronically decay beauty and charm hadrons. The alignment, tracking and particle identification performance will be discussed.

The 2011 Europhysics Conference on High Energy Physics, EPS-HEP 2011,

July 21-27, 2011

Grenoble, Rhône-Alpes, France

\footnotetext{
*Speaker.

${ }^{\dagger}$ On behalf of the LHCb collaboration
} 


\section{Introduction}

The LHCb experiment [1] at the Large Hadron Collider (LHC) at CERN has been designed for precision studies of $\mathrm{CP}$ violation and rare decays of beauty and charm hadrons. Because the $b \bar{b}$ pairs are predominantly produced either in the forward or backward direction, the $\mathrm{LHCb}$ detector is a single arm forward angle spectrometer with coverage of the pseudo-rapidity range of $1.9<\eta<4.9$. Essential features of the $\mathrm{LHCb}$ detector are a vertex detector with an excellent impact parameter and proper time resolution, a tracking system with a good momentum resolution, a powerful particle identification system, and a highly flexible trigger for particle selection. Figure 11 shows a schematic view of the LHCb detector. It consists of a silicon micro-strip Vertex Locator (VELO), followed

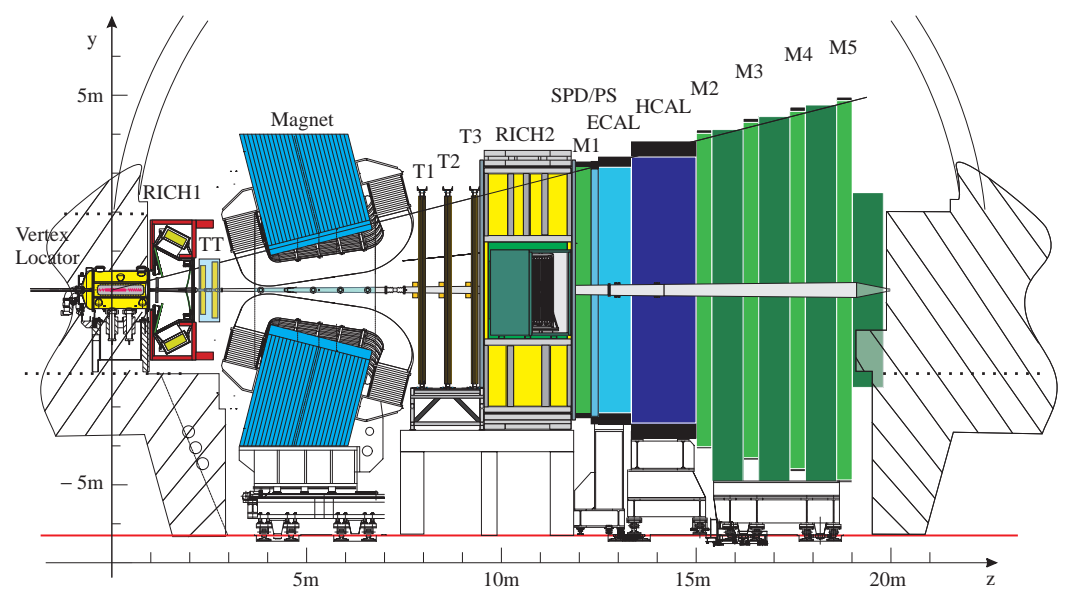

Figure 1: Schematic overview of the LHCb detector.

by the first Ring Imaging Cherenkov counter (RICH), and a silicon strip tracking stations (TT) in front of the (empty) dipole magnet. Behind the magnet there are three layers of tracking stations consisting of a combination of silicon strips and straw-tube drift chambers, a second RICH detector, the electromagnetic and hadronic calorimeter, and finally five muon stations. Further details of the sub-detectors can be found in [1].

\section{Running conditions and trigger}

During the early 2011 data taking, the number of colliding bunches with a spacing of $50 \mathrm{~ns}$ steadily increased to 1296, which is about half of the final number of bunches in the LHC machine. Even with this lower number of bunches LHCb takes data at almost twice its design luminosity, i.e. at $L=3.5 \cdot 10^{32} \mathrm{~cm}^{-2} \mathrm{~s}^{-1}$, thanks to stronger focusing of the beams in the interaction region. This implies that the average number of visible interactions per bunch crossing $\mu$ is 1.5 which is almost a factor four higher than by design. The event reconstruction in the Event Filter Farm proved to operate reliably at this much higher $\mu$ value. Up to July 2011, the LHC delivered $>450 \mathrm{pb}^{-1}$, of which more than $90 \%$ has been recorded by LHCb. With the current LHC filling scheme, LHCb sees about 15 Million bunch crossings per second with a least one interaction. The first level hardware trigger (L0) selects events containing hadrons with a high transverse energy $\left(\mathrm{E}_{t}>3.8 \mathrm{GeV}\right)$ 


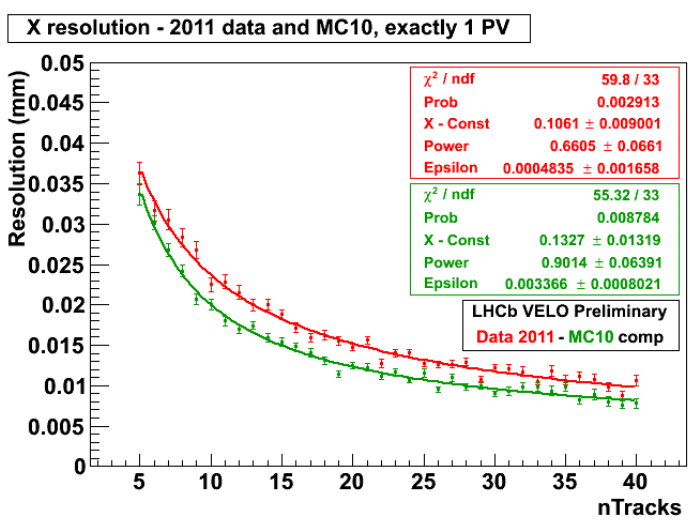

(a)

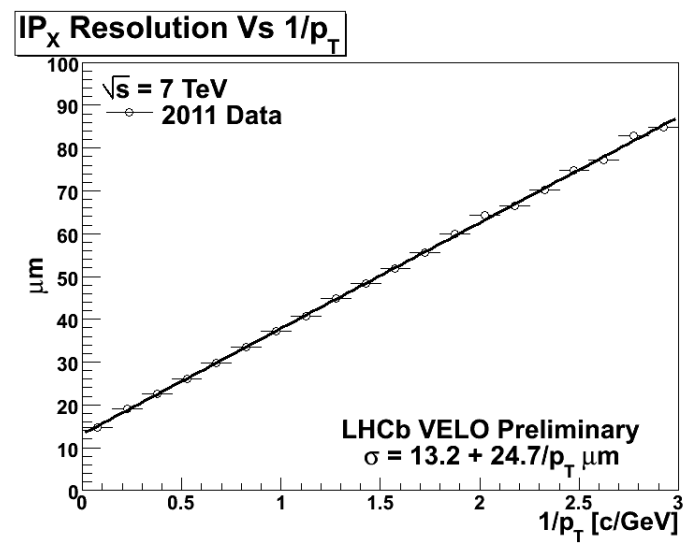

(b)

Figure 2: Left: Primary vertex resolution in the transverse plane as function of the number of tracks. Right: Impact parameter resolution in the transverse plane as function of $1 / \mathrm{p}_{t}$.

detected by the calorimeter system or events with one or more muons with a high transverse momentum $\left(\mathrm{p}_{t}>1 \mathrm{GeV} / \mathrm{c}\right)$ selected by our muon detector. Currently the L0-trigger rate is about $800 \mathrm{kHz}$ (max. $1.1 \mathrm{MHz}$ by design). For every L0 trigger the full detector is read-out and the events are sent to our Event Filter Farm consisting of > 14000 CPU cores which execute the High Level Trigger (HLT) algorithms within an average time budget of $27 \mathrm{~ms}$. The first stage of the HLT further reduces the event rate to $50 \mathrm{kHz}$ by partial event reconstruction and selecting those events which have at least one track with an Impact Parameter (IP) $>100 \mu \mathrm{m}$ and a $\mathrm{p}_{t}$ of $>1 \mathrm{GeV}$. In the second stage, the HLT performs full reconstruction and selects events with a displayed vertex and sufficient mass. In addition dedicated trigger lines are implemented for specific decays. The output rate of the HLT is $3 \mathrm{kHz}$.

\section{Tracking and alignment}

The two main requirements of the tracking system are a very good momentum resolution in order to obtain a good mass resolution, and accurate vertexing which is needed to distinguish between primary and decay vertices and to resolve the fast $\mathrm{B}_{s}$ oscillations. The silicon micro-strip vertex detector achieves a single hit resolution of $4 \mu \mathrm{m}$ in the direction transverse to the beam for the smallest pitch region and optimal angle. A primary vertex resolution of about $12 \mu \mathrm{m}$ in $\mathrm{X} / \mathrm{Y}$ and $65 \mu \mathrm{m}$ in $\mathrm{Z}$ has been obtained for 30-35 track vertices which is the average number of tracks per primary vertex, see left plot of Fig. 2. The corresponding proper-time resolution of about $50 \mathrm{fs}$ allows for an accurate determination of the B-lifetimes (of about $1500 \mathrm{fs}$ ).

Another important performance figure for a B-physics experiment is the impact parameter resolution which for the 2011 data sample is found to be $13.2+24.7 / \mathrm{p}_{t} \mu \mathrm{m}$, see right side of Fig. 2 . The silicon tracking layers in front of, and the silicon + straw-tube layers behind, the $4 \mathrm{Tm}$ magnet provide a momentum resolution of 0.3 to $0.5 \%$. Continuous improvements in the alignment of the tracking stations during the 2010 data taking have resulted in significant improvements in the mass resolution, resulting in for example a $J / \Psi$ mass resolution of $13 \mathrm{MeV} / \mathrm{c}^{2}$. The VELO is a moveable 
detector which is centered around the beam for every fill of the LHC. The stability of the VELO alignment was found to be better than $5 \mu \mathrm{m}$ by determining the difference of the alignment of the two VELO halves separately.

\section{Particle Identification}

LHCb owes its excellent performance to the powerful particle identification systems, which consist of two RICH detectors, an electromagnetic (ECAL) and a hadronic (HCAL) calorimeter, and five muon stations. The first $\mathrm{RICH}$ detector has a dual radiator consisting of aerogel and $\mathrm{C}_{4} \mathrm{~F}_{10}$ and covers a momentum range of $1 \mathrm{GeV} / \mathrm{c}$ up to $60 \mathrm{GeV} / \mathrm{c}$. The second $\mathrm{RICH}$ detector which is located downstream of the magnet covers the high momentum range up to $100 \mathrm{GeV} / \mathrm{c}$ and uses $\mathrm{CF}_{4}$ as detection medium. Angular resolutions of 1.62 and $0.62 \mathrm{mrad}$ have been achieved for the RICH1 and RICH2, respectively. These numbers are very close the values obtained from MC simulations. The efficiency and purity of $\pi / K$ identification over the entire momentum range is shown in the left plot of Fig. 目.

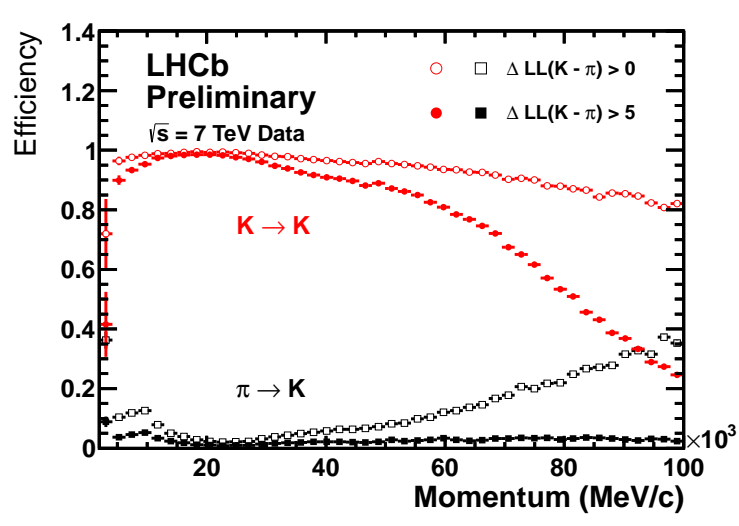

(a)

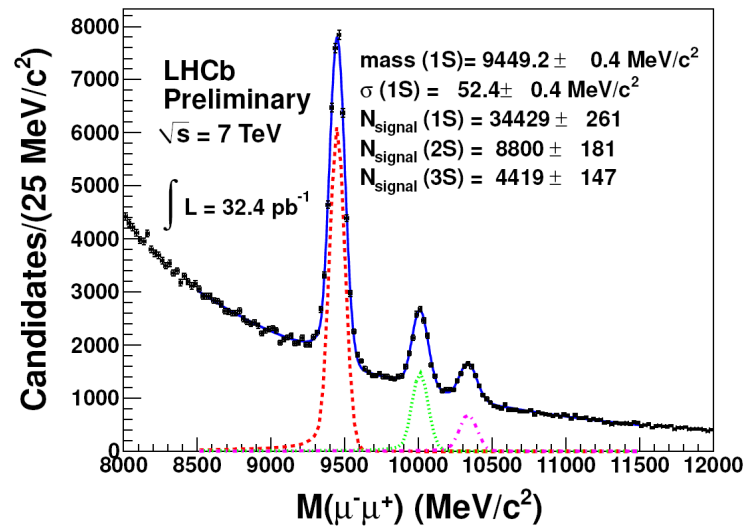

(b)

Figure 3: Left: Efficiency and purity of the Ring Imaging Cherenkov system. Right: Invariant mass of the $\Upsilon(1 S), \Upsilon(2 S)$ and $\Upsilon(3 S)$ resonances.

The ECAL is a shashlik type calorimeter with 66 layers of $2 \mathrm{~mm}$ lead and $4 \mathrm{~mm}$ scintillation material giving an energy resolution of $\sigma / E=\frac{9 \%}{\sqrt{E}} \oplus 0.8 \%$. The HCAL is a sandwich of iron and scintillator material amounting to a total of $5.6 \lambda_{i}$, with a resolution of $\sigma / E=\frac{69 \%}{\sqrt{E}} \oplus 9 \%$. A preshower detector consisting of a $15 \mathrm{~mm}$ lead converter $\left(2.5 \mathrm{X}_{0}\right)$ provides the longitudinal segmentation of the electromagnetic shower detection. Four of the muon stations are positioned behind the HCAL and are interleaved with $80 \mathrm{~cm}$ iron walls. They use two layers of double gap Multi-Wire-Proportional-Chambers. The first station of the muon system is placed in front of the calorimeters and uses because of the high rate triple Gas-Electron-Multiplier technology. Based on a sample of $J / \Psi \rightarrow \mu^{+} \mu^{-}$decays the efficiency has been determined at $97.3 \pm 1.2 \%$ which is in close agreement with the $\mathrm{MC}$ results. The right side of Fig. B gives an example of the performance; shown is the invariant mass of the $\Upsilon(1 S), \Upsilon(2 S)$ and $\Upsilon(3 S)$ resonances. 


\section{Summary}

The $\mathrm{LHCb}$ detector is operating extremely well at almost twice the luminosity it was designed for, and with only half the number of bunches in the LHC. Among the detector performances are an excellent vertex, impact parameter and mass resolution, and powerful particle identification capabilities. Already quite a few world's best measurements in various decay channels have been obtained with this detector and a number of these are shown elsewhere in these proceedings.

\section{References}

[1] A. A. Alves et al. [ LHCb Collaboration ], The LHCb Detector at the LHC JINST 3 (2008) S08005. 